\title{
Fluid fluid levels in bone tumors and tumoral lesions - Pictorial essay \\ Subbarao Kakarla ${ }^{1, *}$ \\ ${ }^{1}$ KIMS Foundation and Research Centre, Minister Road, Secunderabad - 500003, Telangana, India
}

\begin{abstract}
Fluid fluid level is an important and a rare finding noted on CT and MRI images of tumors and tumoral lesions of bone. Although this finding is commonly seen in primary aneurysmal bone cysts but also noted in other benign and malignant neoplasms of bone. Similar finding also is noted in some tumoral lesions, such as, simple bone cyst and infective conditions. The radiological findings of the host lesions in secondary aneurysmal bone cysts as well as other lesions with fluid fluid levels are described to find out whether differentiation can be done in the neoplasms of bone and also from tumoral lesions.
\end{abstract}

Keywords: fluid fluid levels in bone; benign neoplasms; malignant neoplasms; tumoral lesions; CT; MRI; F-FDG PET-CT

\begin{abstract}
*Corresponding author: Prof. Kakarla Subbarao, MS, D.Sc. (HON), FRCR, FACR, FICP, FSASMA, FCCP, FICR, FCGP, Chairman, KIMS Foundation and Research Centre, Minister Road, Secunderabad 500003, Telangana, India. Email: subbaraokakarla25@gmail.com

Received 02 August 2017; Accepted 11 September 2017; Published 18 September 2017

Citation: Kakarla S. Fluid fluid levels in bone tumors and tumoral lesions - Pictorial essay. J Med Sci Res. 2017; 5(4):128-134. DOI: http://dx.doi.org/10.17727/JMSR.2017/5-24

Copyright: @ 2017 Kakarla S, et al. Published by KIMS Foundation and Research Center. This is an open-access article distributed under the terms of the Creative Commons Attribution License, which permits unrestricted use, distribution, and reproduction in any medium, provided the original author and source are credited.
\end{abstract}

\section{Introduction}

The aim of this article is to assess the importance of fluid fluid levels observed on CT and MRI scans and also to know the futility and significance of the fluid fluid levels in determining the underlying pathology in benign, malignant and tumoral lesions without the help of histology. The entities where fluid fluid levels are noted have been analysed. In routine radiological practice, particularly in the interpretation of musculoskeletal bone tumors, fluid fluid levels (ffl) in the images are often encountered in MRI and CT investigations. Their presence is nonspecific. Ffls are only an imageological finding in bone lesions. These are encountered in cystic lesions where there are different density tissues are present in CT and MRI images, in axial/ sagittal slices, in the dependent position. This phenomenon is encountered in hemorrhagic and pyogenic lesions of bone as well as in necrotic tissues of the bone neoplasm irrespective of the histological nature. In portions of blood filled 
lesions, separation of blood and serum takes place in tissue fluids and produce ffls. It is also referred sometimes as liquid liquid levels.

\section{Review of the literature and discussion}

Tsai et al. [1], have reported that ffls not only occur in aneurysmal bone cysts but in other bone and soft tissue neoplasms as well. They conclude that the finding of ffls in bone is not specific for any particular tumor. Van Dyck et al. [2], have extensively reported about the prevalence, extension and characteristic of ffls in bone and soft tissue lesions. However, our report consists of mostly bone lesions. There is only one soft tissue malignant fibrous histiocytoma where there were ffls. Gaillard et al. [3], have described extensively about the ffls in benign as well as in malignant neoplasms of bone. Keenan et al. [4], have published a pictorial essay of musculoskeletal lesions with ffls. Chetan Anchan [5], Hong et al. [6], and
Stefano Boriani [7] published case reports of giant cell tumors with secondary aneurysmal bone cysts with a soft tissue extension and ffls. Kato Tomoyuki et al. [8], found ffls in secondary aneurysmal bone cyst in chondroblastoma of the patella. Ji Seon Park et al. [9], described ffls in haemophilic pseudo tumors also. This finding has taken importance as giant cell tumor is a common benign tumor. The finding of ffls in a bone lesion is very important but not characteristic of any particular lesion of the bone. In our experience a wide variety of lesions of neoplastic and nonneoplastic nature is noted. The characteristic imaging findings correlated with clinical findings and underlying nature of the bone may render correct diagnosis which should be confirmed by histology. The types of bone lesions that demonstrate ffls are described in Table 1. The imageological characteristics of each entity are described with illustrative examples.

Table 1: Bone lesions that can show fluid fluid levels.

\begin{tabular}{|ll|}
\hline Benign tumors - Giant cell tumor & - Chondroblastoma \\
& - Osteoblastoma \\
& - Chondro myxoid fibroma \\
& - Fibrous dysplasia \\
& - Fibroxanthoma \\
& - Hemangioma \\
Malignant bone tumors - Telangiectatic osteosarcoma & - Plasmacytoma \\
& - Malignant fibrous histiocytoma of bone/ soft tissue \\
Tumoral lesions - Aneurysmal bone cyst (ABC) & - Metastasis \\
& - Simple bone cyst (SBC) \\
& - Brodie's abscess \\
& - Brown tumors \\
& - Pseudo tumor of haemophilia.
\end{tabular}

\section{Benign tumors}

\section{Giant cell tumor (GCT) with ABC}

A secondary $\mathrm{ABC}$ develops in association with other neoplasms, most commonly giant cell tumor (GCT) of bone, chondroblastoma, osteoblastoma, fibrous dysplasia etc. Secondary ABC-like changes are seen in $10-14 \%$ of GCTs. When ABC occurs with a GCT of bone, the diagnosis is usually based only on histopathology. However, if solid portion of the image is identified imageologically along with $\mathrm{ABC}$, a diagnosis of secondary ABC in GCT can be suspected. There may be confusion regarding the diagnosis if tissue for biopsy is taken from this cystic region.
However, the presence of $\mathrm{ABC}$ has no implications as far as the management or the outcome of treatment of GCT of bone is concerned.

Giant cell tumor of the bone is a common benign tumor occurring in $3^{\text {rd }}$ and $4^{\text {th }}$ decades. It is metaphyseal lesion extending to the articular margin in a mature skeleton. Radiologically, an osteolytic lesion in the subarticular area is noted. Generally eccentric but expansion occurs with a wide zone of transition indicating the aggressive nature. The soap bubble appearance is common (Figure 1). 

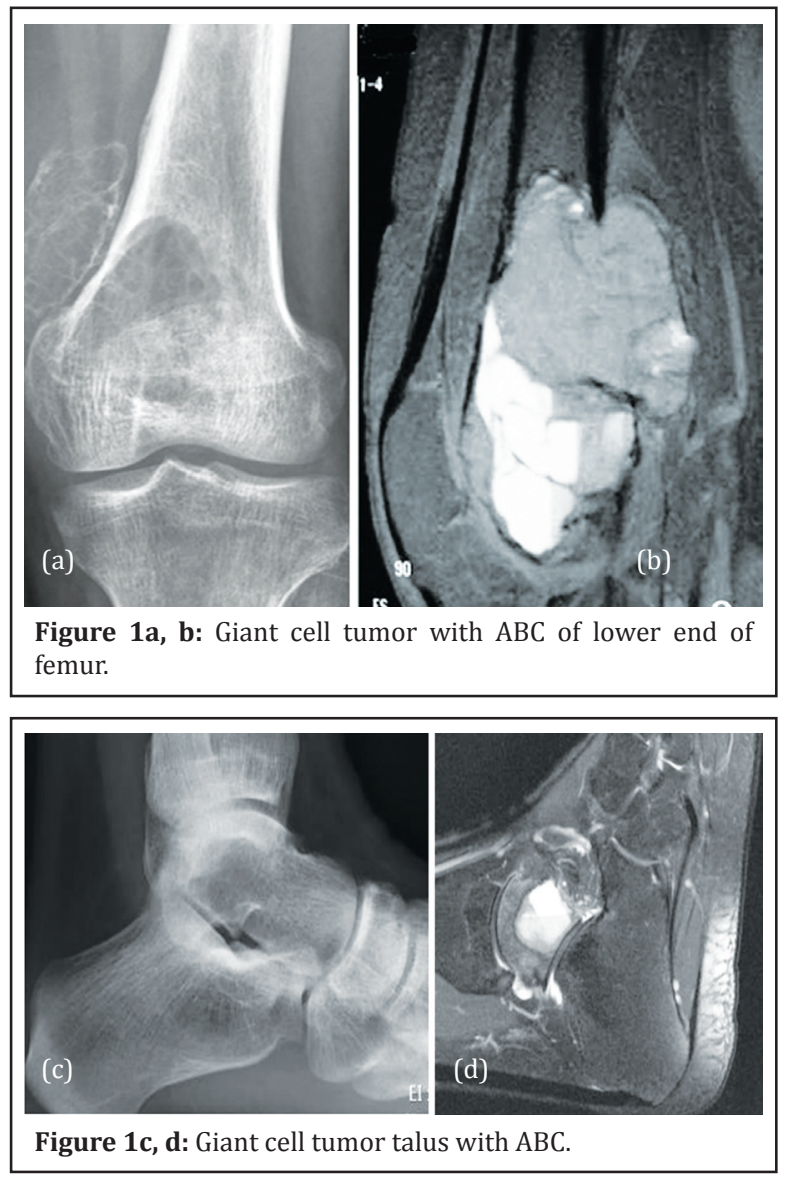

\section{Chondroblastoma with ABC}

Chondroblastoma coexists with $\mathrm{ABC}$ in $10-15 \%$ of the patients. Chondroblastoma comprises $1-3 \%$ of primary bone tumors, occurring in patients between 10 and 20 years of age, and is more prevalent in males [9]. Chondroblastoma is the second common benign bone tumor entertaining secondary aneurysmal bone cysts. Secondary ABC is diagnosed by recognising and analysing the solid underlying lesion. Chondroblastoma is a cartilaginous tumor primarily arising from the epiphysis/ apophysis and may extend to metaphysis. It is commonly noted in second and third decades. Radiologically, a lytic area is noted in the ossification centre of long bones and may extend to the metaphysis. It is well demarcated with a narrow zone of transition. Associated aneurysmal bone cyst occurs in about $15 \%$ of the cases [7] (Figure 2). Chondroid calcification which is often present is characteristic of chondroblastoma.

Osteoblastoma and chondromyxoid fibroma may contain secondary $\mathrm{ABC}$ but there are no radiological characteristics.

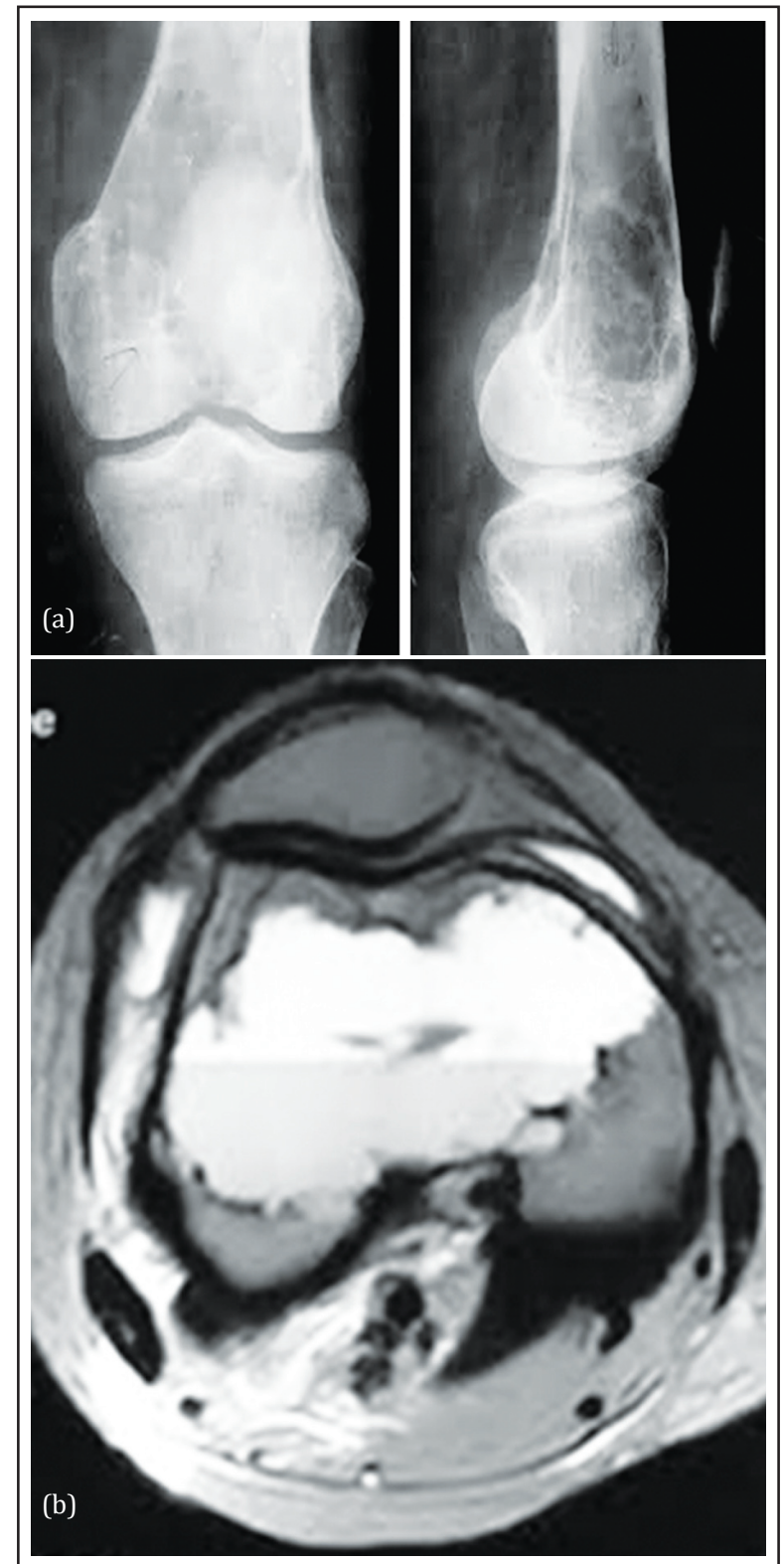

Figure 2a, b: Chondroblastoma with secondary ABC.

\section{Fibrous dysplasia with secondary ABC}

Fibrous dysplasia is a common skeletal dysplasias which can be monostotic, polyostotic or with McCune - Albright syndrome. In some of the phases of cystic degeneration a superimposed $A B C$ can be observed. This is more common in long bones. MRI denotes the secondary ABC. Whereas, fibrous dysplasia can be diagnosed on plain films cystic changes can be noted only on MRI (Figure 3).

Primary malignant bone tumors with $A B C$ : Few malignant lesions can have fluid fluid levels due to necrosis and hemorrhage of the neoplasm. Most 


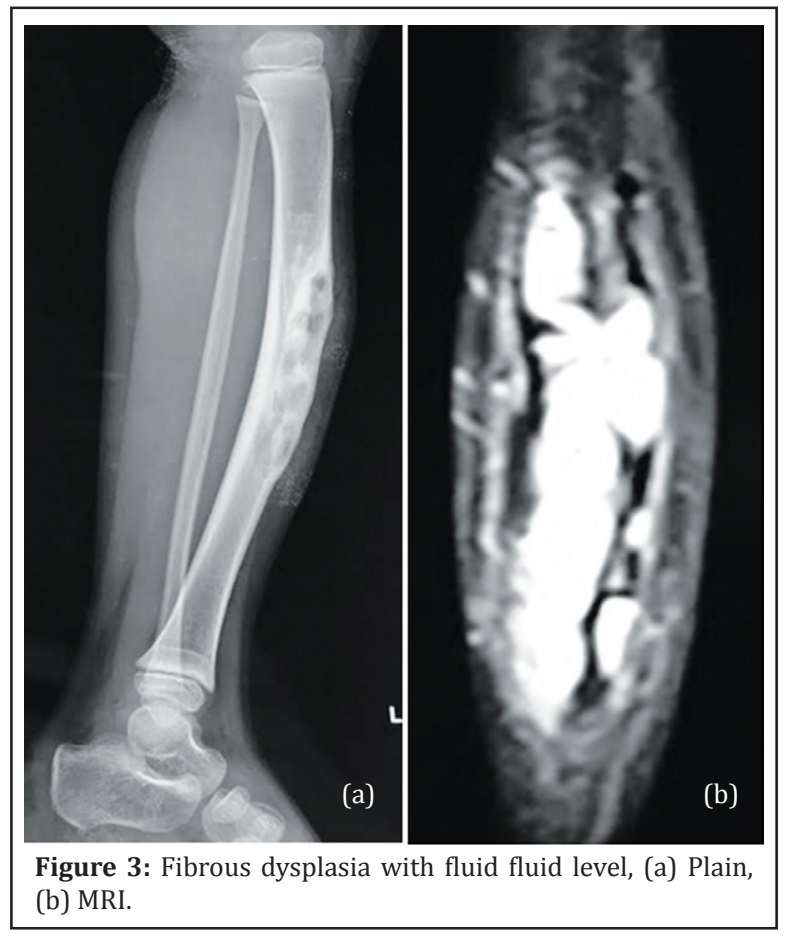

of these malignant tumors are sarcomas including telangiectatic osteosarcoma and chondrosarcoma.

Telangiectatic osteosarcoma is one of the several varities of osteosarcoma which occurs in the same age group, mainly in the long bones. This is a most aggressive type of sarcoma with rapid destruction of bone and very little reactive bone on plain films. It involves cortex and medulla with a large soft tissue swelling. Many large blood filled spaces suggesting ABC (Figure 4a-f).

Chondrosarcoma with fluid fluid levels: Chondrosarcoma is malignant cartilaginous tumor which may be primary or secondary. Primary arises from the central medulla with a lytic lesion containing calcifications. Secondarychondrosarcoma arises from peripherally situated enchondroma or osteochondroma. The imagiological diagnosis of chondrosarcoma include a lytic lesion with a soft tissue mass containing nodular, circular or arc like chondroid calcifications. When cystic or myxomatous degeneration occurs fluid fluid levels may be seen. This is a rare phenomenon (Figure $5 \mathrm{a}, \mathrm{b}$ ).

\section{Plasmacytoma with fluid fluid levels}

Plasmacytoma of bone is a localised manifestation of multiple myeloma. Radiologically an expanding lytic lesion with a wide zone of transition is noted.

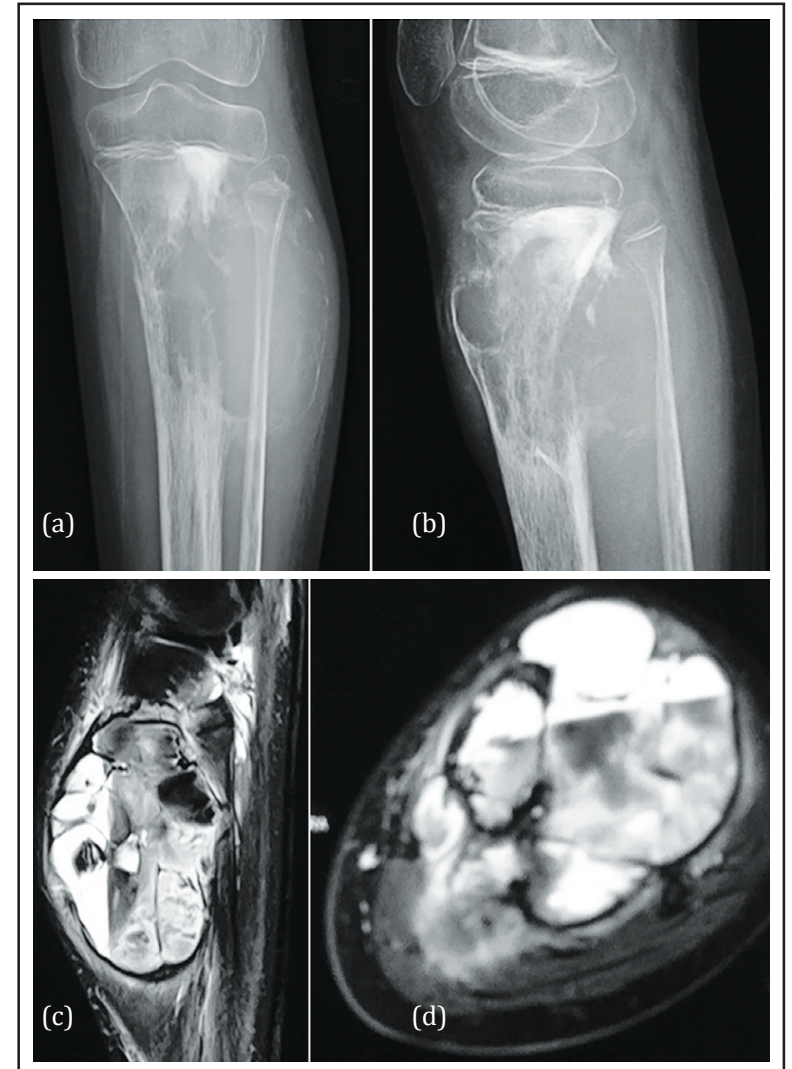

Figure 4a-d: Telangiectatic osteosarcoma with FFL.

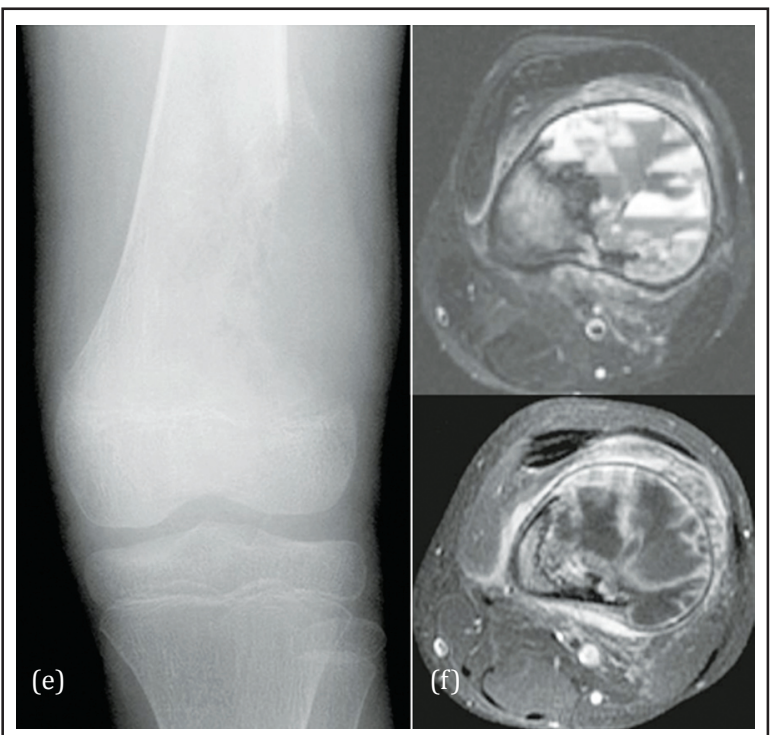

Figure 4e, f: Telangiectatic osteosarcoma with fluid fluid levels.

Soft tissue mass may be large (Figure 6a, b). When the leion contains a great deal of necrotic tissue/ haemorrhage fluid fluid levels are noted. It is rare phenomenon and not frequently reported in the literature. Malignant fibrous histiocytoma of soft tissues with ffls (Figure 7). 

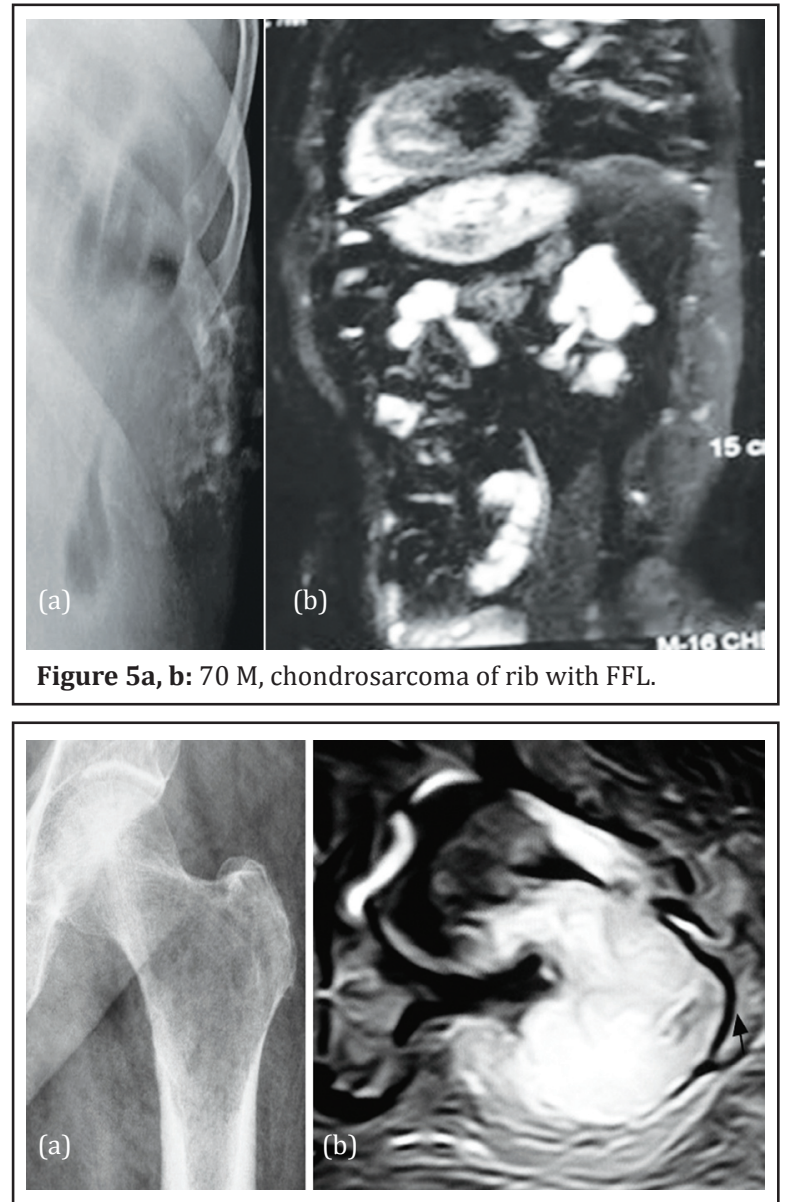

Figure 6a, b: Plasmacytoma with FFL.

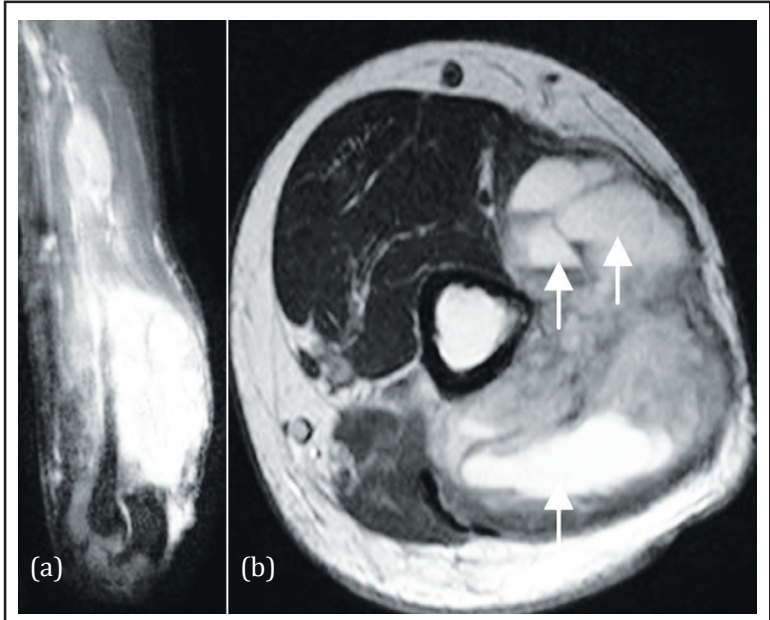

Figure 7a, b: MFH of soft tissues of the arm with ffls (arrows).

Metastatic carcinoma with fluid fluid levels: Blow out skeletal metastasis are common in primary malignancies arising from kidney, breast and thyroid in women, whereas kidney, lung are common in males. Radiologically, a large lytic lesion is noted with MRI showing fluid fluid levels (Figure 8).

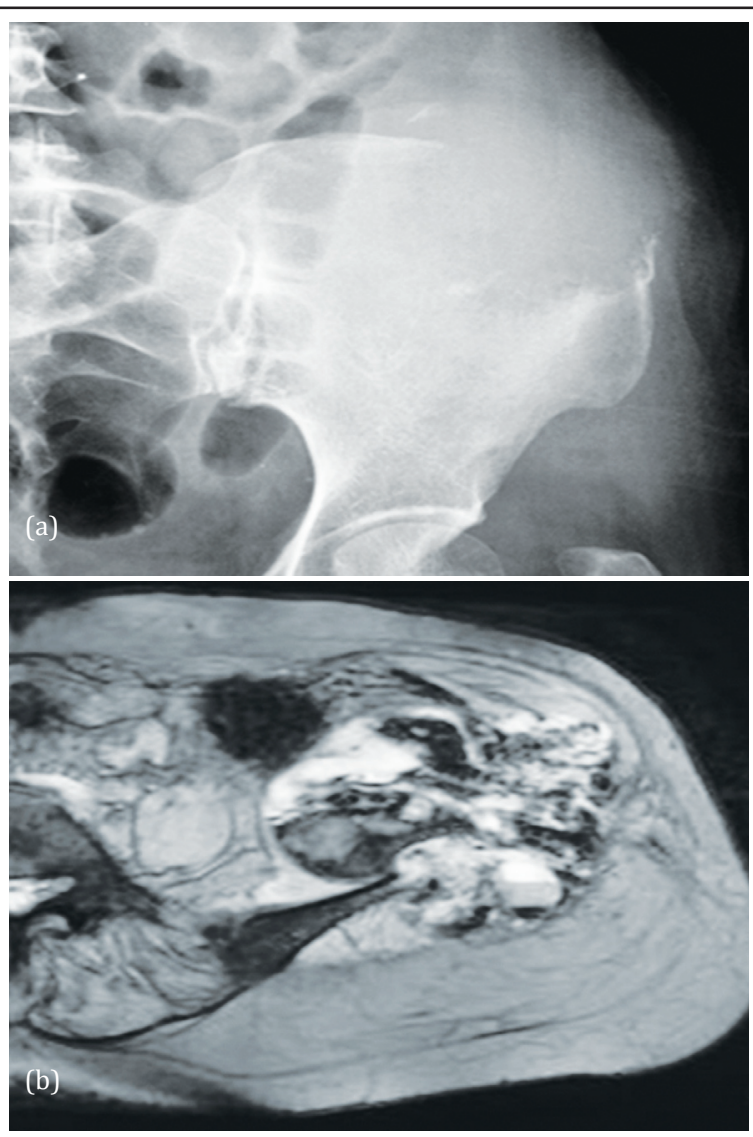

Figure 8a, b: Metastatic carcinoma of carcinoma cervix with fluid fluid levels.

\section{Tumoral lesions with fluid fluid levels Primary aneurysmal bone cyst}

Aneurysmal bone cyst $(\mathrm{ABC})$ is a benign cystic lesion of bone that is composed of blood-filled spaces separated by connective tissue septa containing fibroblasts, osteoclast-type giant cells, and reactive woven bone. Ffls are observed on cross-sectional imaging with either computed tomography (CT) or magnetic resonance (MR) imaging. They become apparent when a fluid collection containing substances of different density is allowed to settle, and when the plane of imaging is perpendicular to the fluid level. Primary ABC can be established only by thorough histological examination. Although when the lesion is occupied entirely by ffls by which a diagnosis of primary $\mathrm{ABC}$ is made it is important to thoroughly scratch the lesion for noting the underlying primary pathology. When this is excluded it can be called primary ABC.

\section{Radiological features of primary ABC}

Radiologically, it is an expanding osteolytic lesion 
with thinning of the cortex and a shell may be observed. The zone of transition is narrow but can be aggressive (Figures $9 \& 10$ ). The lesion may cross the growth plate in children. Fluid fluid levels may be shown on CT/MRI. Although solitary aneurysmal bone cysts exist. They are often seen at below the age of $20 \mathrm{yrs}$.
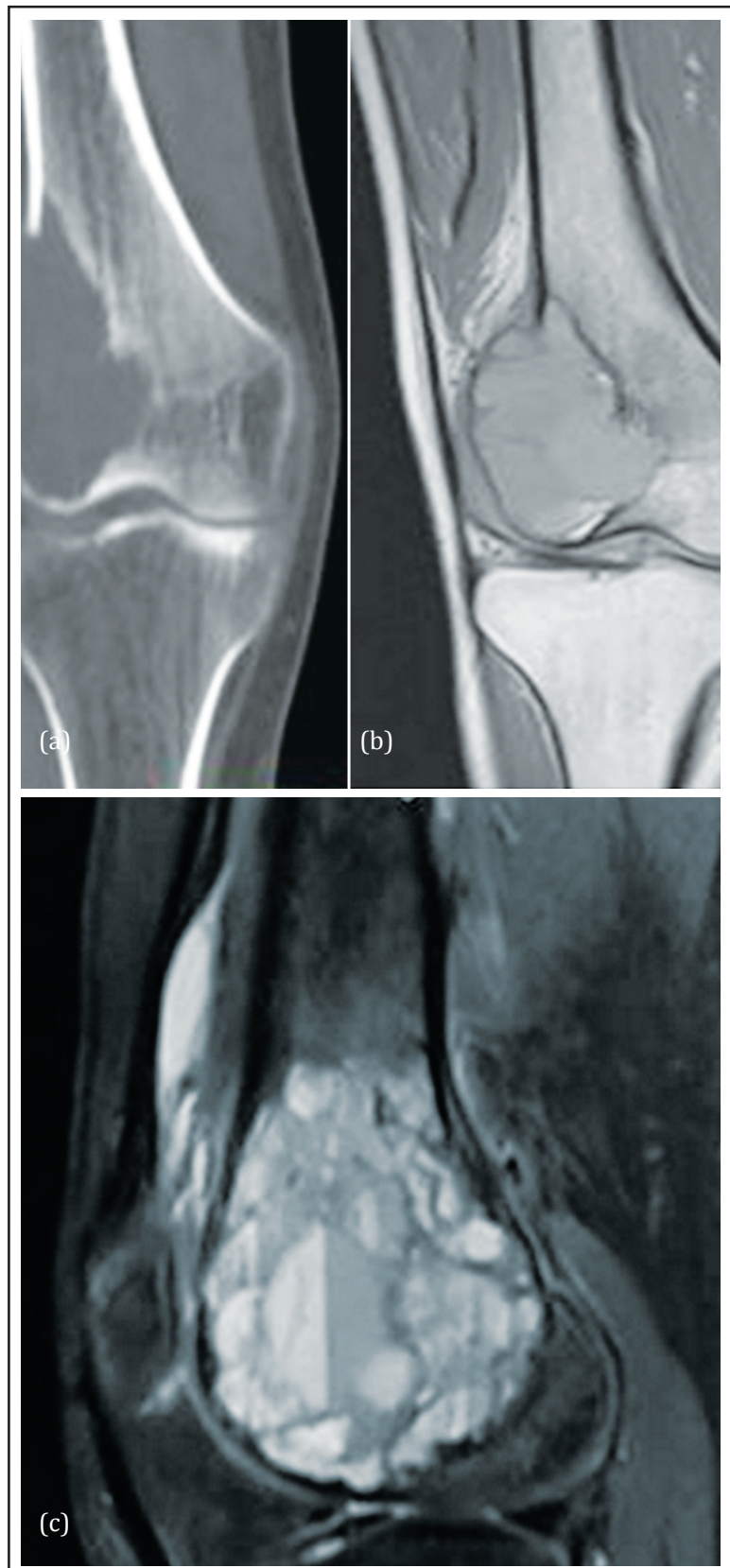

Figure 9a, b, c: 19 yrs - Primary aneurysmal bone cyst in the lower end of femur (Note the multiple ffls).

\section{Simple bone cyst (SBC)}

Most common tumoral lesion noted in the $1^{\text {st }}$ two decades of life. Common location is proximal

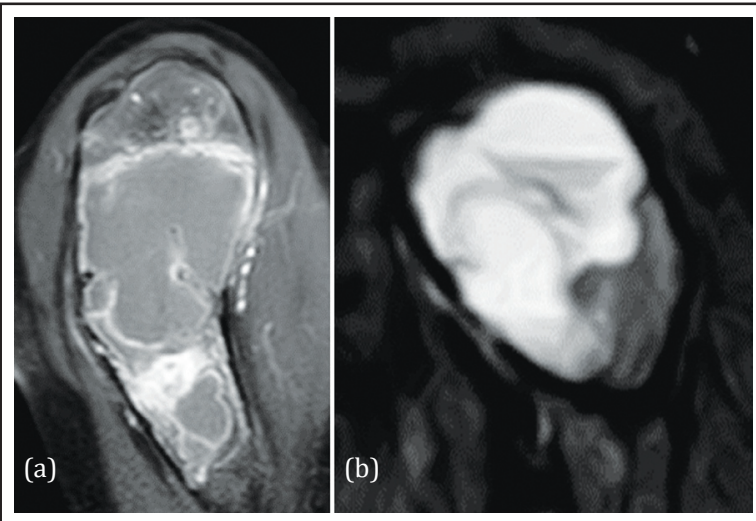

Figure 10a, b: Primary ABC with ffl.

metaphysis of humerus. Radiologically, an expanding osteolytic lesion is noted in the metaphyseal region. The overlying cortex of the thinned and a narrow transitional zone is noted. The multilocular appearance is due residual cortical edges. Films obtained with a horizontal beam may demonstrate a fallen fragment following a fracture. Although metaphyseal to begin with may extend to diaphysis. MRI may show ffls (Figure 11a, b).

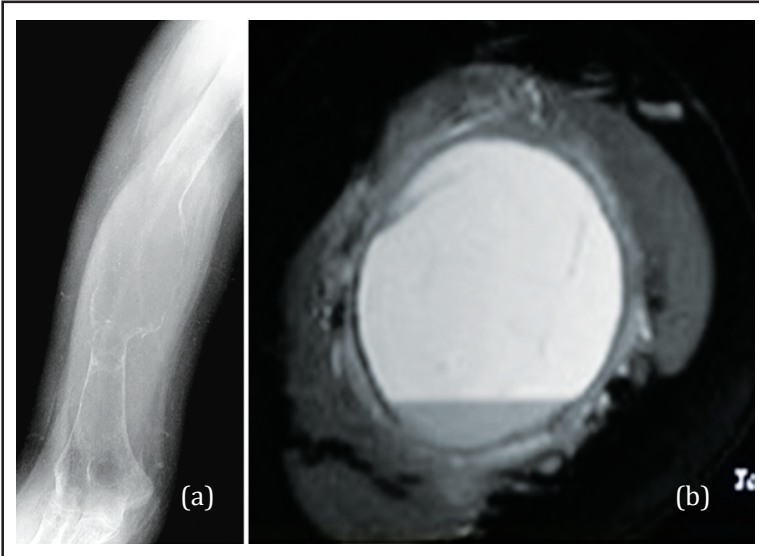

Figure 11a, b: 24 F Simple bone cyst with ffls.

Brodie's abscess with ffls: Despite extensive usage of antibiotics in fighting infections, osteomyelitis is still encountered in children and adolescents. Osteomyelitis is a radiological spectrum with acute, subacute and chronic stages. In subacute stage a localised abscess may be noted mostly located in the metaphyseal lesion. Radiologically, a lucent area of various sizes is noted with a sclerotic rim. Occasionally, periosteal reaction also may be present. Further imaging is not usually necessary. MRI shows ffls with surrounding high attenuated area. This may have low attenuated rim called penumbra (Figure 12). 


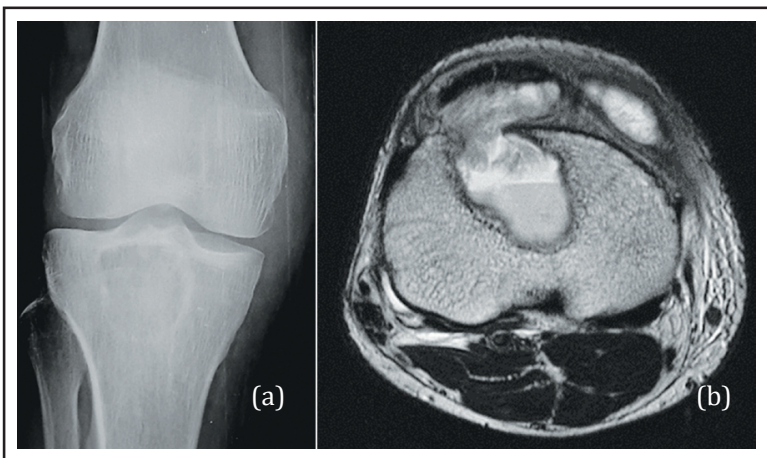

Figure 12a, b: Brodie's abscess with ffls on MRI.

Brown tumors with ffls: Brown tumors of bone may be noted in primary or secondary hyperparathyroidism. These are localised areas of collection of unmineralised osteoblasts with spindle cells and variable amounts of haemorrhages. On plain films these look like benign tumors of bone due to haemorrhages multiple ffls may be noted on MRI (Figure 13a, b).

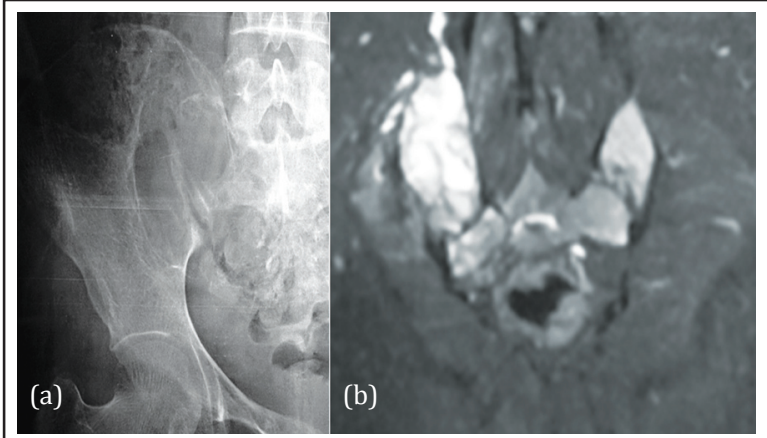

Figure 13a, b: 26F, Brown tumor of hyperparathyroidism

Hemophilic pseudo tumor with ffls: Bleeding in to the bone in hemophilic patients is often called pseudo tumor of haemophilia. On MRI ffls may be noted due to degenerative products of blood. In the involvement of pseudo tumor, ffls have been described. However, we didn't find any example of this in our files. The other entities included in the list are also rare.

\section{Conclusion}

This article is to describe ffls found on CT and MRI scans in various lesions of bone and soft tissues, which include benign and malignant tumors as well as tumor like lesions. The final conclusion is this finding of ffls does not help in determining the original nature of the lesion except in primary aneurysmal bone cysts and few other tumors. These lesions also should be correlated with clinical, imageological and histological findings. Whenever ffls are noted, it is important to study the primary lesion histologically.

\section{Acknowledgements}

NIMS, KIMS, KREST Museum Hyderabad.

\section{Conflicts of interest}

Author declares no conflicts of interest.

\section{References}

[1] Tsai JC, Dalinka MK, Fallon MD, Zlatkin MB, Kressel HY. Fluidfluid level: a nonspecific finding in tumors of bone and soft tissue. Radiology. 1990; 175(3):779-782.

[2] Van Dyck P, Vanhoenacker FM, Vogel J, Venstermans C, Kroon $\mathrm{HM}$, et al. Prevalence, extension and characteristics of fluidfluid levels in bone and soft tissue tumors. Eur Radiol. 2006; 16(12):2644-2651.

[3] Frank Gaillard A, et al., Fluid-fluid level containing bone lesions; Radiopaedia. Available from: https://radiopaedia. org/articles/fluid-fluid-level-containing-bone-lesions-2.

[4] Keenan S, Bui-Mansfield LT. Musculoskeletal lesions with fluid-fluid level: a pictorial essay. J Comput Assist Tomogr. 2006; 30(3):517-524.

[5] Chetan Anchan. Giant cell tumor of bone with secondary aneurysmal bone cyst; Int J Shoulder Surg. 2008; 2(3):68.

[6] Hong WS, Sung MS, Kim JH, Kim HM, Kim TK, et al. Giant cell tumor with secondary aneurysmal bone cyst: a unique presentation with an ossified extraosseous soft tissue mass. Skeletal Radiol. 2013; 42(11):1605-1610.

[7] Boriani S, Bandiera S, Ghermandi R, Colangeli S, Amendola L, et al. Aneurysmal Bone Cyst and Giant Cell Tumor. Musculoskeletal Key. 2016; Available from: https:// musculoskeletalkey.com/aneurysmal-bone-cyst-and-giantcell-tumor/

[8] Tomoyuki K, Susa M, Nakayama R, Watanabe I, Horiuchi $\mathrm{K}$, et al. Secondary aneurysmal bone cyst following chondroblastoma of the patella. Rare Tumors. 2013; 5(3):e43.

[9] Park JS, Ryu KN. Pseudotumor Involving the Musculoskeletal System: Spectrum of Radiologic Findings. American Journal of Roentgenology. 2004; 183:55-61. 